CMS RPC commissioning of the existing detector during the long shutdown

This content has been downloaded from IOPscience. Please scroll down to see the full text. 2014 JINST 9 C10043

(http://iopscience.iop.org/1748-0221/9/10/C10043)

View the table of contents for this issue, or go to the journal homepage for more

Download details:

IP Address: 157.193.98.234

This content was downloaded on 11/06/2015 at 14:01

Please note that terms and conditions apply. 


\section{CMS RPC commissioning of the existing detector during the long shutdown}

A. Cimmino, ${ }^{f, 1}$ Y. Ban, ${ }^{a}$ J. Cai, ${ }^{a}{ }^{\text {Q. Li, }},{ }^{a}$ S. Liu, ${ }^{a}$ S. Qian, ${ }^{a}$ D. Wang,${ }^{a}$ Z. Xu, ${ }^{a}$ F. Zhang, ${ }^{a}$ Y. Choi ${ }_{,}^{b}$ D. Kim, ${ }^{b}$ J. Goh ${ }^{b}$ S. Choi, ${ }^{c}$ B. Hong, ${ }^{c}$ J.W. Kang, ${ }^{c}$ M. Kang, ${ }^{c}$ J.H. Kwon, ${ }^{c}$ K.S. Lee, ${ }^{c}$ S.K. Lee, ${ }^{c}$ S.K. Park, ${ }^{c}$ L.M. Pant, ${ }^{d}$ A.K. Mohanty, ${ }^{d}$ R. Chudasama, ${ }^{d}$ J.B. Singh, ${ }^{e}$ V. Bhatnagar, ${ }^{e}$ A. Mehta, ${ }^{e}$ R. Kumar, ${ }^{e}$ S. Cauwenbergh,${ }^{f}$ S. Costantini, ${ }^{f}$ S. Crucy, ${ }^{f}$ A. Fagot, ${ }^{f}$ G. Garcia, ${ }^{f}$ A. Ocampo, ${ }^{f}$ D. Poyraz,${ }^{f}$ S. Salva,${ }^{f}$ F. Thyssen, ${ }^{f}$ M. Tytgat,${ }^{f}$ N. Zaganidis, ${ }^{f}$ W.V. Doninck, ${ }^{g}$ A. Cabrera, ${ }^{h}$ L. Chaparro, ${ }^{h}$ J.P. Gomez, ${ }^{h}$ B. Gomez, ${ }^{h}$ J.C. Sanabria, ${ }^{h}$ C. Avila, ${ }^{h}$ A. Ahmad, ${ }^{i}$ S. Muhammad, ${ }^{i}$ M. Shoaib,${ }^{i}$ H. Hoorani, ${ }^{i}$ I. Awan, ${ }^{i}$ I. Ali, ${ }^{i}$ W. Ahmed,${ }^{i}$ M.I. Asghar, ${ }^{i}$ H. Shahzad, ${ }^{i}$ A. Sayed,${ }^{j}$ A. Ibrahim, ${ }^{j}$ S. Aly, ${ }^{j}$ Y. Assran, ${ }^{j}$ A. Radi, ${ }^{j}$ T. Elkafrawy, ${ }^{j}$ A. Sharma, ${ }^{k}$ S. Colafranceschi, ${ }^{k}$ M. Abbrescia, ${ }^{l}$ C. Calabria, ${ }^{l}$ A. Colaleo, ${ }^{l}$ G. laselli, ${ }^{l}$ F. Loddo, ${ }^{l}$ M. Maggi, ${ }^{l}$ S. Nuzzo, ${ }^{l}$ G. Pugliese, ${ }^{l}$ R. Radogna, ${ }^{l}$ R. Venditti, ${ }^{l}$ P. Verwillingen, ${ }^{l}$ L. Benussi, ${ }^{m}$ S. Bianco, ${ }^{m}$ D. Piccolo, ${ }^{m}$ P. Paolucci, ${ }^{n}$ S. Buontempo, ${ }^{n}$ N. Cavallo, ${ }^{n}$ M. Merola, ${ }^{n}$ F. Fabozzi, ${ }^{n}$ O.M. Iorio, ${ }^{n}$ A. Braghieri, ${ }^{o}$ P. Montagna,${ }^{o}$ C. Riccardi, ${ }^{o}$ P. Salvini, ${ }^{o}$ P. Vitulo, ${ }^{o}$ I. Vai, ${ }^{o}$ A. Magnani, ${ }^{o}$ A. Dimitrov,${ }^{p}$ L. Litov, ${ }^{p}$ B. Pavlov, ${ }^{p}$ P. Petkov, ${ }^{p}$ A. Aleksandrov, ${ }^{q}$ V. Genchev, ${ }^{q}$ P. laydjiev, ${ }^{q}$ M. Rodozov, ${ }^{q}$ G. Sultanov, ${ }^{q}$ M. Vutova, ${ }^{q}$ S. Stoykova, ${ }^{q}$ R. Hadjiiska, ${ }^{q}$ H.S. Ibargüen, ${ }^{r}$ M.I.P. Morales, ${ }^{r}$ S.C. Bernardino, ${ }^{r}$ I. Bagaturia, ${ }^{s}$ Z. Tsamalaidze, ${ }^{s}$ I. Crotty ${ }^{t}$ and M.S. Kim ${ }^{u}$ on behalf of the CMS collaboration

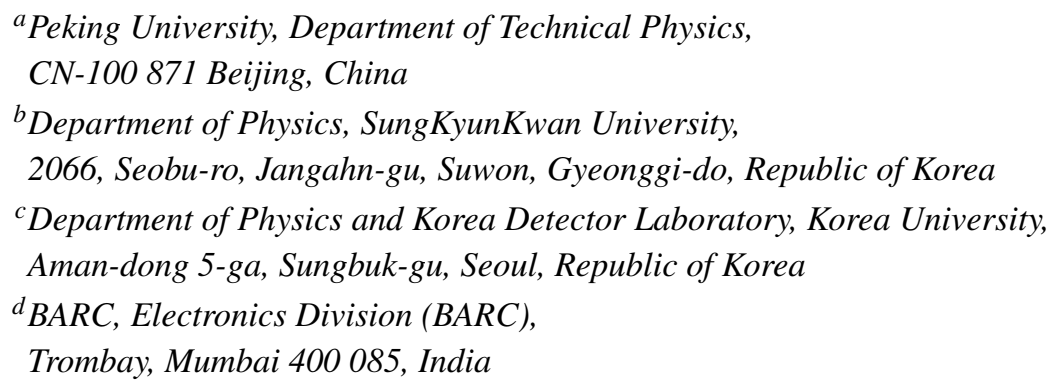

${ }^{1}$ Corresponding author 
${ }^{e}$ Panjab University, Department of Physics,

Chandigarh Mandir 160 014, India

${ }^{f}$ Ghent University, Department of Physics and Astronomy,

Proeftuinstraat 86, BE-9000 Ghent, Belgium

${ }^{g} V U B$, Dienst Elementaire Deeltjes,

Pleinlaan 2 BE-1050 Brussel

${ }^{h}$ Universidad de Los Andes,

Apartado Aéreo 4976, Carrera 1E, no. 18A 10, CO-Bogotá, Colombia

${ }^{i}$ Islamabad-NCP, Quaid-I-Azam University,

Islamabad-44000 Pakistan

${ }^{j}$ Academy of Scientific Research and Technology of the Arab Republic of Egypt,

101 Sharia Kasr El-Ain, Cairo, Egypt

${ }^{k} C E R N$,

CH-1211 Geneva 23

${ }^{l}$ Universita e INFN, Sezione di Bari,

Via Orabona 4, IT-70126 Bari, Italy

${ }^{m}$ INFN, Laboratori Nazionali di Frascati, PO Box 13,

Via Enrico Fermi 40, IT-00044 Frascati, Italy

${ }^{n}$ Universita e INFN, Sezione di Napoli, Complesso Univ. Monte S. Angelo,

Via Cintia, IT-80126 Napoli, Italy

${ }^{o}$ Universita e INFN, Sezione di Pavia,

Via Bassi 6, IT-Pavia, Italy

${ }^{p}$ University of Sofia, Faculty of Physics, Atomic Physics Department,

5, James Bourchier Boulevard, BG-1164 Sofia, Bulgaria

${ }^{q}$ Bulgarian Academy of Sciences, Inst. for Nucl. Res. and Nucl. Energy,

Tzarigradsko shaussee Boulevard 72, BG-1784 Sofia, Bulgaria

${ }^{r}$ Benemérita Universidad Autónoma de Puebla,

Av. San Claudio y 18 sur, edif. 111 A Ciudad Universitaria,

Col. San Manuel, Puebla, Pue. C.P. 72570, Mexico

${ }^{s}$ Tibilisi-IHEPI, Tbilisi State University University,

Street 9 GE-380 086 Tbilisi

${ }^{t}$ University of Wisconsin, Department of Physics,

1150 University Avenue Madison, WI 53706 U.S.A.

${ }^{u}$ Kyungpook National University, Department of Physics,

80 Daehakro, Bukgu, Daego, 702-701, Republic of Korea

E-mail: anna.cimmino@cern.ch 
ABSTRACT: February $14^{\text {th }} 2013$ marked the end of the first period of running of the Large Hadron Collider (LHC) and the start of a two-year break from operation (LS1) aimed at consolidating both the accelerator as well as the detectors. By the end of LS1, the LHC is expected to provide collisions at $13 \mathrm{Tev}$. While, by 2020, the ultimate instantaneous luminosity is expected to be $10^{34} / \mathrm{cm}^{2} / \mathrm{s}$. To prepare for this scenario, the Resistive Plate Chamber system at the CMS experiment is planning several detector maintainance and consolidation interventions. These include High Voltage and Low Voltage system reparations, gas leak identification and reparation, signal channel connectivity and functionality. Commissioning and upgrade plans for the existing CMS RPC system are presented here.

KEYWORDS: Gaseous detectors; Particle tracking detectors (Gaseous detectors) 


\section{Contents}

1 Introduction 1

2 RPC commissioning and upgrade during LS1 2

$2.1 \mathrm{HV}$ and LV interventions 2

2.2 Gas leak campaign 3

2.3 Signal channel connectivity and functionality test 3

3 Conclusions $\quad 4$

\section{Introduction}

The first running period of the Large Hadron Collider (LHC) came to a stop in February 2013, effectively starting the first long shutdown, LS1. Refurbishment and consolidation of both the accelerator system and the detectors has started and will last until 2015. At the end of LS1, the LHC is expected to provide collisions at a center-of-mass energy of $13 \mathrm{TeV}$. In addition, the expected separation between successive collisions at this energy will reach the nominal $25 \mathrm{~ns}$. While, by 2020 , the ultimate instantaneous luminosity is expected to be $10^{34} / \mathrm{cm}^{2} / \mathrm{s}$. To prepare for this scenario, the CMS [1] collaboration planned several detector upgrades [2]. In particular, the present Resistive Plate Chamber (RPC) [3] system, which serves as a dedicated muon triggering system, will be enlarged with the addition of a fourth layer in the endcap regions on either sides of the detector [4]. Such an extension is necessary to maintain a low- $\mathrm{p}_{T}$ threshold for an efficient Level-1 Muon Trigger at high particle rates. These new endcap stations are equipped in the region $0.9<|\eta| \leq 1.6$ with 144 double-gap RPCs with bakelite plates, operating in avalanche mode. But upgrading the existing detector is just one side of the medallion. Consolidating and commissioning the existing system is equally important to ensure high performance throughout the lifetime of the experiment and to cope with increasing collision energy and instantaneous luminosity.

RPCs at CMS [5] form a massive system, extending well over $3000 \mathrm{~m}^{2}$ with a pseudo-rapidity coverage of $|\eta| \leq 1.6$. This sums up to double-gap 912 chambers, 2316 eta-partitions, 109608 strips, 696 High Voltage (HV) channels, 1152 Low Voltage (LV) channels, 1232 Link Boards (LB) housed in 96 crates, 401 temperature sensors, and approximately $16 \mathrm{~m}^{3}$ of Freon-based gas mixture, LV boards and LBs are mounted in racks around the detector in the experimental hall. The HV system is located in the auxiliary cavern housing the counting room and the technical services, along with the trigger boards. The gas bottles, purifiers, and mixtures are instead located in a surface building. While hundreds of kilometers of cables, tubes, and optic fibers interconnect all parts. Access to the experimental hall is possible only during LHC downtime and must be carefully planned and documented. Material leaving the experimental hall, even is only temporarily, must pass through the underground buffer zone for scanning, logging in the material database, and appropriate labeling for traceability. It is, therefore, clear that interventions on the system during run-periods are extremely complicated and long shutdown periods represent unique opportunities. 


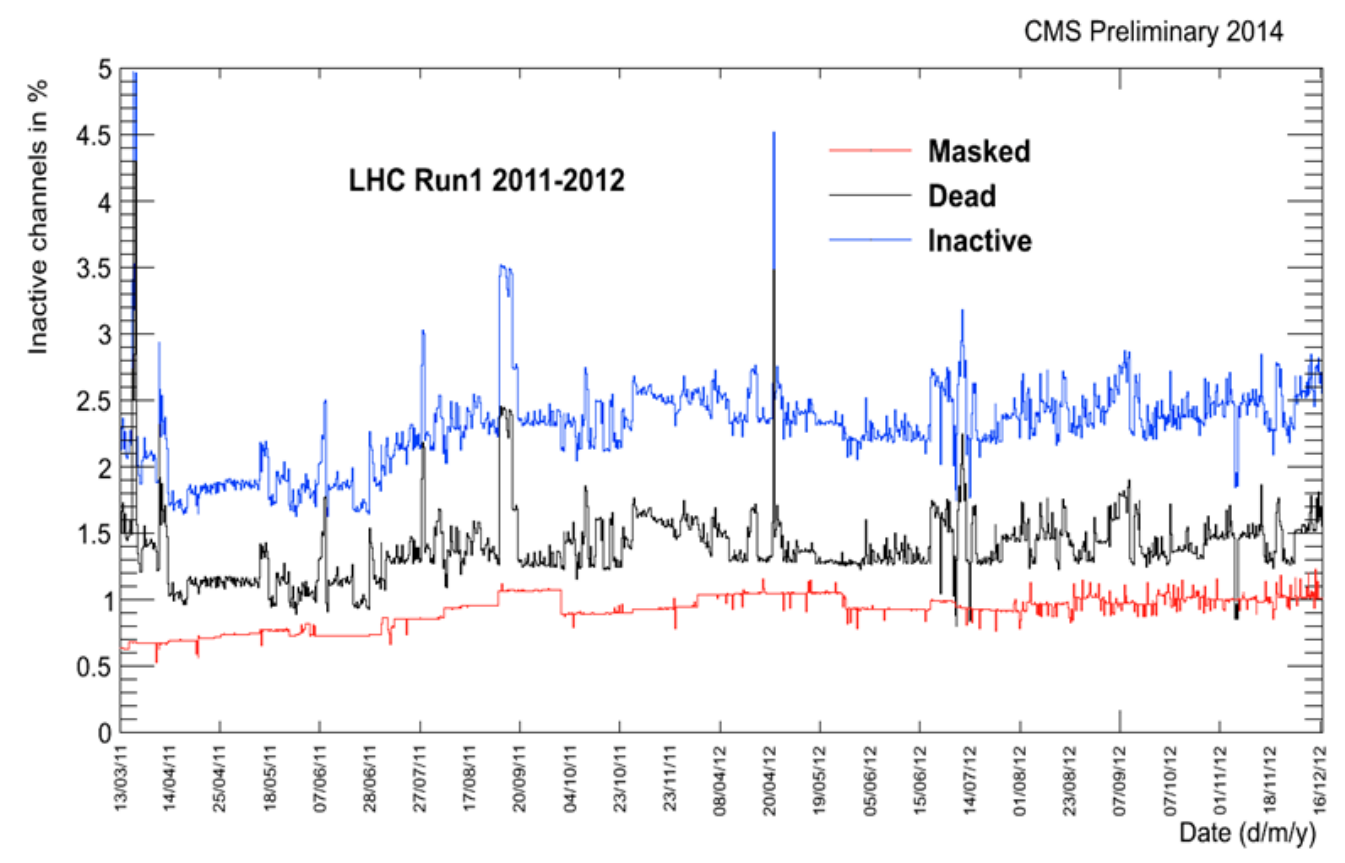

Figure 1. History of the percentage of dead or masked channels during proton-proton collision runs in 2012. The total percentage of dead and masked channels combined, which are called inactive channels, is also shown in the plot.

\section{RPC commissioning and upgrade during LS1}

During LS1, commissioning and upgrade interventions scheduled by the RPC system may be classified as follows: High Voltage (HV) and Low Voltage (LV) systems reparation, gas leak estimation and reduction, signal channel connectivity and functionality, and software upgrade. Technical interventions have been scheduled to recover 15 disconnected chambers and the $2 \%$ of inactive electronic channels. A gas leak survey and power systems checks are being performed to completely review of the system.

\subsection{HV and LV interventions}

After three years of running, the RPCs at CMS participated to more than 2200 collisions runs and were responsible of as little as $0.6 \%$ of luminosity loss, collecting a total integrated luminosity of $\sim 29 \mathrm{fb}^{-1}$. At the start of LS1, active channel were measured to be $\sim 98 \%$, as it may be seen in figure 1. Recovering the largest number of inactive channels, due to HV or LV problems, is extremely important during LS1 to maintain these high performances. In fact, not all parts of the detector may be accessible during future long shutdowns. By February 2014, the RPC community, intervened on $55 \%$ of the system and recovered $1.11 \%$ of all electronic channels. HV problems were mostly due to broken connectors that were promptly replaced. LV problem, instead, often required extraction of the chamber and replacement of the front-end board. Repaired channels are extensively tested. Current and voltage stability is monitored via the Detector Control System (DCS) [6]. An intuitive Graphical User Interface (GUI) eases this commissioning task significantly. 
Plots, diagrams, histograms, and tables are available for a prompt online analysis of the detector behavior. Finally, all data collected by the DCS, is stored in the Online Master Data Storage (OMDS) [7] for offline analysis and performance studies.

\subsection{Gas leak campaign}

The RPC gas mixture is non-flammable and made of $95.2 \% \mathrm{C}_{2} \mathrm{H}_{2} \mathrm{~F}_{4}$ (Freon), $4.5 \% i \mathrm{C}_{4} \mathrm{H}_{10}$ (isobutene), and $0.3 \% \mathrm{SF}_{6}$ (sulfur hexafluoride). The humidity of the mixture is set at $45 \%$ to keep bakelite resistivity constant $\left(1-2 \times 10^{10} \Omega \mathrm{cm}\right)$. A closed-loop circulation system [8], was developed by the CERN gas group because of the high costs and huge volumes of this Freon-based gas mixture. The closed-loop system consists of several modules distributed among various locations in the experimental area. On the surface, the gas is mixed at the proper concentration, humidified, analyzed. Then, it is pumped to the gas distribution racks, located around the detector in the underground experimental hall. The mixture is subsequently distributed to the different stations and the relative flows are constantly monitored in order to detect possible leaks. The outputted gas is cleaned from impurities and put back in recirculation with a fresh injection of gas limited to $10 \%$. Tightness of this system is important not only for the safe operation of the detector, but also for environmental and economical reasons. A systematic gas leak survey and reparation campaign is being conducted [9]. By fluxing the chambers with Argon, the total gas leak was, at the start of LS1, $\sim 644 \mathrm{l} / \mathrm{h}$ and entirely located in the barrel region $(0 \leq|\eta|<1.2)$. Figure 2 shows examples of gas pressure difference measurements versus time and serves as a template for classifying chamber behavior. Once the problematic chambers are identified, $\mathrm{N}_{2} \mathrm{H}_{2}$ is fluxed through them. Using sniffers, leaks were localized and repaired where possible. At the time of this paper, $60 \%$ of the barrel was inspected and the leak was reduced by $51 \%$. The majority of the leaks were due to broken T-connectors within the chambers. The stress and strain applied on these plastic junctions by the same gas tubes they connect causes them to crack.

\subsection{Signal channel connectivity and functionality test}

The primary purpose of this test is to ensure the integrity of the cabling. The second goal is to avoid swaps between neighboring channels. To allow for interventions, in fact, bundles of cables are disconnected from each chambers and swaps or loose connections are bound to occur. A fully automated tool is in place to check all connections and allows fixes while access is still available. This is done by varying Front End Board (FEB) threshold level and reading out systematically each channel. The tool, written entirely in $\mathrm{C}++$, was developed in XDAQ (Cross-Platform DAQ Framework) [10], a software platform designed at CERN specifically for the development of distributed data acquisition systems. Configuration, control, and monitoring of the RPC FEBs are developed in XDAQ. Figure 3 shows the presence of a swapped cable between neighboring partitions of the same chamber, clearly noticeable by the empty bins in the left plot, which refers to the backward partition. The expected signal, instead, appears in the corresponding bins of the right plot which refers to the forward partition. Reparation, in this case, was straightforward once the chamber in question was made accessible. Channel connectivity and functionality tests are also performed on all newly installed chambers in the fourth endcap stations. 


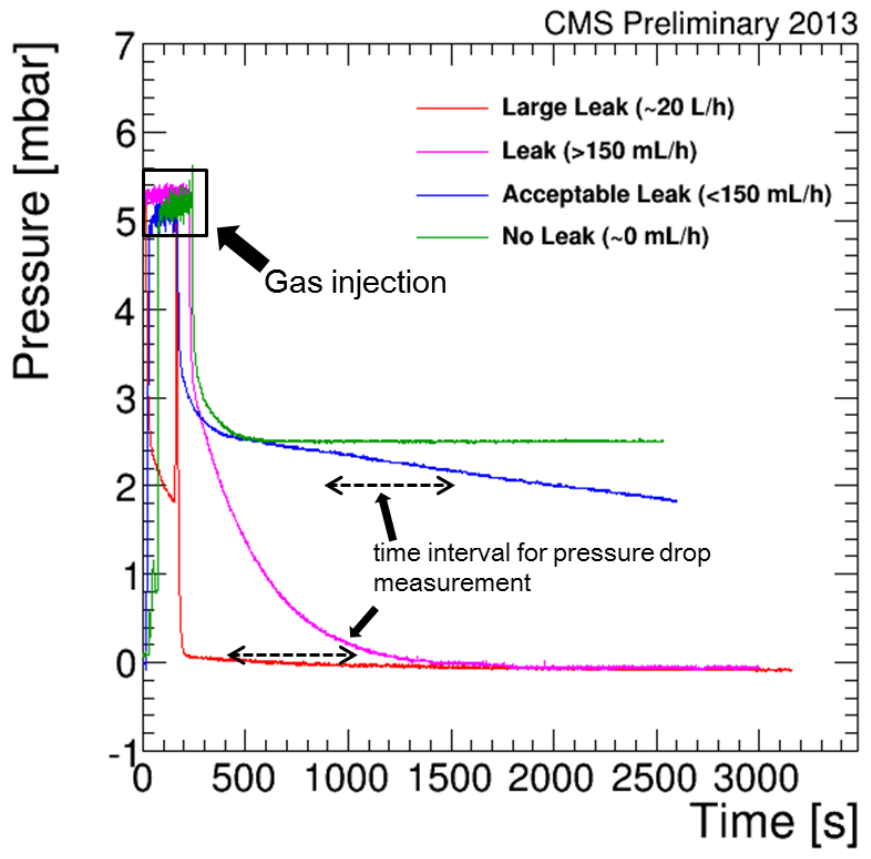

Figure 2. This figure shows four different examples of RPC gas pressure difference as a function of time. These are the raw data with a special tool, called gas leak box, supported by the CERN gas group. The green line shows a stable leak. The blue line shows an acceptable leak. The magenta line shows a bigger leak than the blue line and a potential problem. Leak rates were calculated after a stabilization period of maximum 10 minutes after gas injection and they were calculated using pressure drop intervals of 10 minutes. Finally, the red line shows a very large leak that exceeds the full scale of the gas leak box. Gas leak rates for chambers showing this behavior were were estimated using the gas input rate.

\section{Conclusions}

As the instantaneous luminosity and center-of-mass energy of the LHC continue to increase, the CMS experiment must ensure stable and efficient operation. In particular, the Muon System should be able to keep its trigger rate and efficiency under control at a low enough muon $\mathrm{p}_{T}$-threshold for physics studies. To this end, the RPC system has been extended with the addition of a $4^{\text {th }}$ layer in both endcap regions. However, consolidating the existing detector is equally important to ensure high performance throughout the lifetime of the experiment and to cope with increasing collision energy and instantaneous luminosity. This two-year break in LHC operation provides the perfect opportunity. A tight schedule of $\mathrm{HV}, \mathrm{LV}$, and Gas systems inspections and reparations was planned and is being carried through along with the installation of the new chambers. At the start of LS1, the number of inactive electronic channel was measured to be $\sim 2 \%$. At present, half-way through LS1, $1.11 \%$ of the electronic channels were repaired. The gas leak survey managed to reduce the leak by $\sim 51 \%$. Signal channel connectivity and functionality is tested after all interventions to ensure the integrity of the cabling. Commissioning activities are still ongoing and will last until 2015. 

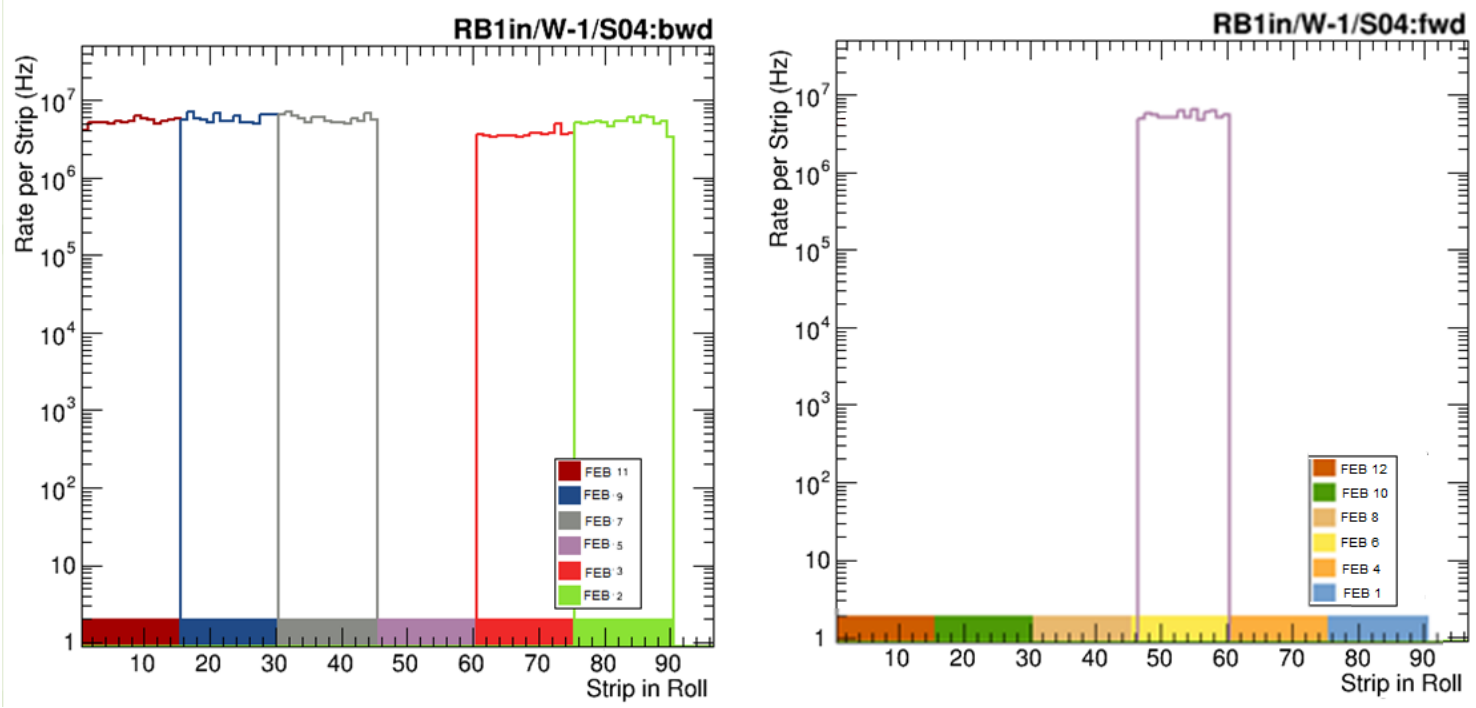

Figure 3. Example result for the connectivity and functionality test on a chamber in the barrel region. The ordinate axis shows the rate per strip. The rate is given in units of $s^{-1}$. A swap between cable in neighboring partitions is noticeable by the empty bins in the left plot, which refers to the backward partition. The expected signal, instead, appears in the corresponding bins of the right plot which refers to the forward partition.

\section{References}

[1] CMS collaboration, The CMS experiment at the CERN LHC, 2008 JINST 3 S08004.

[2] CMS collaboration, Technical proposal for the upgrade of the CMS detector through 2020, CERN-LHCC-2011-006.

[3] R. Santonico and R. Cardarelli, Development of Resistive Plate Counters, Nucl. Instrum. Meth. 187 (1981) 377.

[4] M. Tytgat et al., The Upgrade of the CMS RPC System during the First LHC Long Shutdown, PoS RPC2012 (2012) 063 [arXiv:1209.1979].

[5] CMS collaboration, The Muon Project, CMS Technical Design Report, CERN/LHCC 97-32, CMS-TDR-003.

[6] G. Polese et al., The detector control systems for the CMS resistive plate chamber, J. Phys. Conf. Ser. 219 (2010) 022019.

[7] M. de Gruttola et al., Persistent storage of non-event data in the CMS databases, 2010 JINST 5 P04003 [arXiv: 1001.1674].

[8] M. Capeans, R. Guida, F. Hahn, S. Haider and B. Mandelli, RPC performances and gas quality in a closed loop gas systemfor the new purifiers configuration at LHC experiments, 2013 JINST 8 T08003.

[9] S. Jiwoong for the CMS collaboration, Optimization of the gas system in the CMS RPC Detector at the LHC, CMS-CR-2013-429 (2013).

[10] V. Brigljevic et al., Using XDAQ in Application Scenarios of the CMS Experiment, CMS-CR-2003-007. 